\title{
Sistema Colaborativo para Captação de Doadores de Sangue
}

\author{
Arthur O. da Silva ${ }^{1}$, Luana L. Rodrigues ${ }^{2}$, Rodrigo R. Oliveira ${ }^{2}$, Rodrigo R. \\ Oliveira $^{3}$ \\ ${ }^{1}$ UFSC - Campus Araranguá, ${ }^{2}$ IFSUL - Campus Sapucaia do Sul, ${ }^{3}$ UFRGS \\ o.arthur.oliveira@grad.ufsc.br, luanaleiter@hotmail.com, \\ remor@sapucaia.ifsul.edu.br, ruasoliveira@inf.ufrgs.br
}

\begin{abstract}
The shortage of donors in blood centers is a major public health problem in Brazil. To tackle this issue, public servants from blood donation centers and the media are constantly working on campaigns to encourage donation. However, a large part of the population remains passive. In this work, we propose a system to help attract donors and non-donors to blood banks. To do this, the system allows blood centers and general population to create events and content and disseminate them through a mobile application and integration with popular social networks.
\end{abstract}

Resumo. A falta constante de doadores de sangue nos hemocentros é um dos grandes problemas da saúde pública no Brasil. Para tentar reverter esse problema, os servidores dos centros de coleta e as midias trabalham em campanhas com o intuito de incentivar a doação voluntária. Entretanto, grande parte da população continua passiva. Neste artigo propõe-se um sistema colaborativo que auxilie a aproximar doadores e não doadores dos hemocentros. Para tanto, o sistema permite que os hemocentros e a população criem eventos e conteúdos e divulguem-os por meio de um aplicativo móvel e integração com redes sociais populares.

\section{Introdução}

A doação de sangue é um problema de interesse mundial, visto que não há como substituir completamente o tecido sanguíneo [Rodrigues et al. 2011] e este é essencial em diversos procedimentos de saúde, como recuperação de acidentes, operações cirúrgicas e tratamento de enfermidades. Por esse motivo, a Organização Mundial da Saúde (OMS) reconhece o uso do sangue, e seus componentes, como essenciais aos sistemas nacionais de assistência à saúde [OMS 2015].

Apesar da importância, no Brasil os hemocentros enfrentam dificuldades em manter os estoques de bolsas de sangue regulares para suprir as demandas [Rodrigues 2013]. A Organização Mundial da Saúde recomenda que cada país tenha entre 3\% a 5\% da população como doadores ativos [OMS 2006]. Entretanto, no Brasil, em 2012, o percentual teve uma média geral de $1,9 \%$ da população. Para piorar, os estoques dos 
hemocentros de todo o país caem em épocas de frio e chuva ou em períodos de férias comuns. A redução média no inverno é de $20 \%$ a $25 \%$, podendo chegar a $40 \%$ nos estados de São Paulo, Rio de Janeiro e Rio Grande do Sul [Ministério da Saúde 2014].

Para que se doe mais sangue é necessário educar, informar, motivar e desfazer mitos e crenças [Giacomini 2007, Gutiérrez et al. 2003]. Para tal, os centros de hemoterapia constantemente realizam campanhas a respeito da doação de sangue [Dani 2009], expondo a população a mensagens sobre o tema em diversos meios. Em particular, as tecnologias sociais representam grande potencial para aumentar o impacto da conscientização, uma vez que permitem a transformação de interesses e valores em normas orientadoras do comportamento humano [Fowler e Christakis 2010, Ludwig 2010]. Todavia, as poucas plataformas colaborativas existentes não oferecem aproximação de fato, pois são orientadas apenas a hemocentros ou apenas a doadores, mas não a ambos. É necessário que a população e os hemocentros, principais atores no processo de captação, atuem de forma integrada e colaborativa na geração dessas atividades. Nossa conjectura é que se os hemocentros e a população trabalharem em conjunto na construção de eventos para gerar conscientização, o sistema terá maior abrangência e manutenibilidade. Com isso, a captação e conservação de doadores será potencialmente maior.

Neste artigo, propõe-se desenvolver um sistema para aproximar doadores e nãodoadores de centros de hemoterapia por meio de uma rede colaborativa integrada aos hemocentros públicos do Brasil. Essa estratégia é genérica e busca-se a construção de um sistema colaborativo de âmbito nacional. Para cumprir o objetivo geral, o sistema busca oferecer:

- Acesso à informação. Desenvolve-se um aplicativo móvel para agilizar e simplificar a busca por informações sobre como se tornar um doador e fornecer respostas para as dúvidas e mitos mais comuns;

- Colaboração. Permite-se que doadores e hemocentros criem eventos, tais como: pedidos de doação para indivíduos ou bancos de sangue, alertas de baixa em estoques ou de alta procura, palestras informativas, dentre outros;

- Divulgação. Viabiliza-se a integração com Facebook e Twitter para a disseminação de informação, campanhas e eventos, aumentando o número de pessoas envolvidas e informadas. Assim, atinge-se tanto usuários participantes quanto usuários não-participantes e indiretos (aqueles que recebem divulgações mesmo sem utilizar ou possuir vínculo com o sistema);

- Manutenção. Visa-se a estimular o uso, manutenção e divulgação da plataforma por meio da liberação da plataforma como código aberto e do emprego de padrões amplamente adotados pela indústria.

\section{Trabalhos relacionados}

Para identificar critérios de comparação relevantes ao desenvolvimento do sistema, foram realizadas duas visitas em hemocentros da região metropolitana do estado do Rio Grande 
do Sul: (1) Hemocentro do Estado do Rio Grande do Sul (HEMORGS) e (2) Serviço de Hemoterapia de Novo Hamburgo (HEMOVIDA). Dois pontos se sobressaíram: a dificuldade na criação e divulgação de ações de incentivo a doação de sangue; e a falta de acesso à informação sobre a doação, por exemplo sobre a localização dos pontos de coleta de sangue e hemocentros da região.

Dessa forma, percebe-se a necessidade de meios que permitam aos hemocentros compartilharem e divulgarem com mais abrangência suas ações de captação de doadores. Em contrapartida, é preciso envolver a população nessas atividades e torná-la mais ativa nesse processo para criar um vínculo de colaboração entre ambos. Também é fundamental educar a população sobre o tema e permitir o acesso às informações relativas ao processo de doação; uma vez que esses são pilares essenciais para a ampliação da captação de doadores [Pinochet 2011, Rodrigues 2013].

Para classificar os sistemas colaborativos atuais baseou-se no modelo $3 \mathrm{C}$ [Pimentel et al. 2003]. Esse conhecido modelo considera que a colaboração envolve: comunicação (troca de mensagens), coordenação (gerenciamento de pessoas, atividades e recursos) e cooperação (operações em um espaço compartilhado para execução de tarefas). A classificação considera quatro características essenciais: acesso à informação (Comunicação), auxílio aos hemocentros (Cooperação), colaboração população/hemocentro (Comunicação e Cooperação), gerência e manutenção de ações de incentivo (Coordenação). Acesso à informação significa oferecer diversas informações de maneira facilitada, por exemplo, sobre a localização dos centros de doação, campanhas e eventos de coletas móveis. Auxílio aos hemocentros compreende facilitar a gerência do hemocentro, oferecendo, por exemplo, controle de intervalo de doações, criação de eventos e coleta de estatísticas. Colaboração população/hemocentro consiste em dar liberdade para que hemocentros, doadores, receptores e familiares possam participar mais ativamente do sistema, por exemplo, criando eventos, solicitando doações direcionadas e notificando (doadores) sobre pedido de doação de forma simples e rápida. Gerência e manutenção de ações de incentivo a doação de sangue visa permitir, aos hemocentros, controlar o acesso ao sistema e a divulgação dos materiais discutidos anteriormente. Espera-se que, combinando essas quatro características, a comunidade cresça de forma autônoma sem depender de alguns poucos administradores.

A Tabela 1 compara trabalhos relacionados qualitativamente, indicando se os mesmos oferecem $(\mathrm{V})$, não oferecem $(\mathrm{N})$, ou oferecem parcialmente $(\mathrm{P})$ as características essenciais listadas acima.

A maior parte das plataformas disponíveis nesse contexto não oferece qualquer tipo de colaboração, manutenção ou acesso à informação. As poucas plataformas colaborativas existentes são Hemoliga [Rodrigues 2015], Hemogram [Instituto das Cidades Inteligentes 2017] e Hemobile [Underline 2016]. Todos oferecem acesso à informação relacionada a doação de sangue, contudo o Hemogram é o aplicativo que exibe o maior número de informações sobre o processo de doação. Essa plataforma, em particular, possibilita que seus usuários façam pedidos de doação e através de um cadastro 
possam receber alertas quando houver a necessidade de doação de sangue conforme o tipo sanguíneo do usuário; porém, não presta auxílio aos hemocentros quanto a criação de campanhas e eventos relativos a doação.

O Hemoliga permite aos usuários acompanhar o estoque atual de sangue em hemocentros que são cadastrados em seu servidor, viabiliza aos centros de coleta a criação de campanhas para doação e possibilita aos usuários a oportunidade de acompanhar campanhas em curso, não oferecendo funcionalidades para a geração de ações de incentivo a doação pelo usuário, como solicitação de doação.

Tabela 1. Comparação de aplicativos relacionados.

\begin{tabular}{|c|c|c|c|c|}
\hline \multirow{2}{*}{ CATEGORIA } & \multirow{2}{*}{$\begin{array}{c}\text { SUB- } \\
\text { CATEGORIA }\end{array}$} & \multicolumn{3}{|c|}{ PLATAFORMAS SEMELHANTES } \\
\hline & & HEMOLIGA & HEMOGRAM & HEMOBILE \\
\hline \multirow{3}{*}{$\begin{array}{l}\text { COOPERAÇÃO (auxílio aos } \\
\text { hemocentros; colaboração } \\
\text { população/hemocentro;) }\end{array}$} & $\begin{array}{l}\text { Lembrete de intervalo } \\
\text { de doação. }\end{array}$ & $(\mathrm{N})$ & (V) & $(\mathrm{V})$ \\
\hline & $\begin{array}{c}\text { Permite aos } \\
\text { hemocentros divulgar } \\
\text { ações de incentivo } \\
\text { oficiais. }\end{array}$ & (V) & $(\mathrm{N})$ & $(\mathrm{N})$ \\
\hline & $\begin{array}{l}\text { Criação de solicitação } \\
\text { de doação de sangue. }\end{array}$ & $(\mathrm{N})$ & $(\mathrm{V})$ & (V) \\
\hline \multirow{5}{*}{$\begin{array}{l}\text { COMUNICAÇÃO (colaboração } \\
\text { população/hemocentro; acesso à } \\
\text { informação) }\end{array}$} & Campanhas. & $\begin{array}{l}\text { (P) Somente } \\
\text { hemocentros } \\
\text { geram } \\
\text { campanhas. }\end{array}$ & $\begin{array}{l}\text { (P) Somente } \\
\text { usuários geram } \\
\text { campanhas. }\end{array}$ & $\begin{array}{l}\text { (P) Somente } \\
\text { usuários geram } \\
\text { campanhas. }\end{array}$ \\
\hline & Lista de hemocentro. & $(\mathrm{V})$ & $(\mathrm{N})$ & $(\mathrm{V})$ \\
\hline & $\begin{array}{l}\text { Mapa com localização } \\
\text { dos hemocentros. }\end{array}$ & (V) & (V) & (V) \\
\hline & $\begin{array}{l}\text { Notificação e } \\
\text { compartilhamento via } \\
\text { redes sociais. }\end{array}$ & $(\mathrm{N})$ & $\begin{array}{c}(\mathrm{V}) \\
\text { Facebook }\end{array}$ & $\begin{array}{l}\text { (V) } \\
\text { Facebook e } \\
\text { Whatsapp }\end{array}$ \\
\hline & Fornece acesso. & (V) & (V) & $(\mathrm{V})$ \\
\hline \multirow{2}{*}{$\begin{array}{l}\text { COORDENAÇÃO (gerência e } \\
\text { manutenção de ações de } \\
\text { incentivo) }\end{array}$} & Cadastro & (V) & (V) & (V) \\
\hline & $\begin{array}{l}\text { Controle de acesso e } \\
\text { manutenção dos } \\
\text { materiais divulgados }\end{array}$ & (V) & $(\mathrm{N})$ & $(\mathrm{N})$ \\
\hline
\end{tabular}

Fonte: desenvolvida pelos autores.

O Hemobile é um aplicativo voltado para a criação de campanhas de doação. Ele permite que os usuários criem suas solicitações e as divulguem para os demais usuários através do compartilhamento destas em redes sociais como o Facebook e o WhatsApp. Além disso, oportuniza a comunicação entre doador e receptor através da criação de grupo de conversa nas campanhas; mas não promove a integração e geração de ações de 
incentivo pelos centros de coleta. De forma a restringir esses eventos aos pedidos de doação criadas pelos usuários da plataforma, ou seja, a manutenção do aplicativo se dá pela ação do usuário, excluindo os hemocentros desse processo e não promovendo a divulgação de suas ações.

Dentre os trabalhos relacionados, é possível verificar que nenhum apresenta uma plataforma que permita a criação e manutenção de ações de incentivo a doação de sangue pelos hemocentros em conjunto com a criação desses eventos pelos usuários. Todas elas dispõem somente uma dessas características, ou seja, há uma limitação quanto aos eventos divulgados e compartilhados nesses sistemas. Dessa forma, reforçamos nossa conjectura de que se hemocentros e população atuarem de forma colaborativa, haverá maior potencial para captação e conservação de doadores.

\section{O sistema}

O sistema proposto utiliza uma plataforma web, um aplicativo mobile e integração com redes sociais para facilitar o acesso, criação e disseminação de conteúdo ligado ao tema. Ele abrange três tipos principais de usuário: servidor de hemocentro, comum (doador ou não-doador) e indireto (que faz uso de redes sociais).

O restante desta seção descreve o sistema de duas formas principais. Primeiro apresenta-se, na Subseção 3.1, uma visão abrangente dos principais componentes e entidades do sistema e as suas possíveis interações. Após, na Subseção 3.2, discute-se os aspectos de implementação de cada componente principal.

\subsection{Visão geral da arquitetura do sistema}

A Figura 1 resume o sistema. O componente App permite que os usuários acessem todos os eventos criados pelos hemocentros (no componente Web), assim como criar suas próprias solicitações de reposição de sangue. Além disso, é possível registrar suas doações de sangue e optar por receber uma notificação quando estiver apto a doar novamente. $\mathrm{O}$ aplicativo também tem o intuito de disponibilizar uma página com informações básicas sobre doação de sangue e um fluxograma sobre a doação para que seja possível sanar dúvidas dos usuários (atualmente fundamentadas em regulamentos, normas e estudos relacionados à captação de sangue [Ministério da Saúde 2016]).

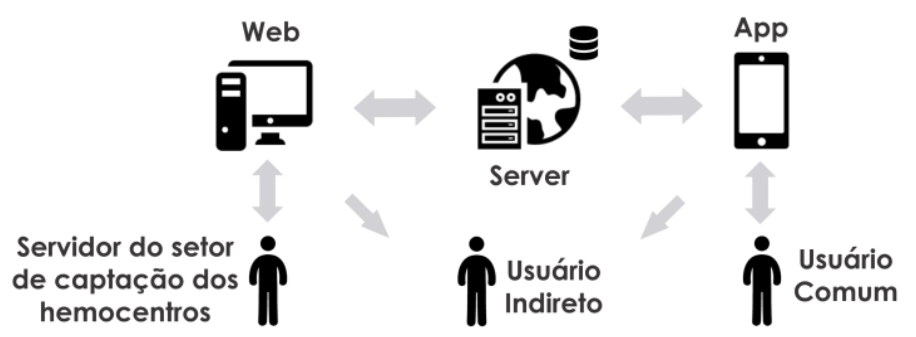

Figura 1. Visão geral do sistema 
O componente Web é uma ferramenta de gerenciamento de ações de captação. Por isso, os usuários do setor de captação dos hemocentros podem utilizá-lo para criar eventos com as informações necessárias para tal, como localidade e categoria (coleta externa, palestra, campanha). Assim que geradas, essas ocorrências também são notificadas aos usuários do componente App e listadas em uma aba de eventos.

O componente Server é o responsável pelas principais funcionalidades do sistema. Ele trata as informações recebidas dos outros componentes e as guarda em uma base de dados. Sua principal tarefa é disponibilizar recursos sob forma de serviços para os componentes Web e App.

Conforme mencionado, o sistema compreende três tipos de usuários: servidor do hemocentro, comum e indireto. O servidor do hemocentro é o administrador do componente $W e b$. Ele pode acessar a conta do hemocentro onde atua e criar, modificar e excluir eventos de diversas categorias relacionadas ao tema (campanhas, solicitações, etc). Ele também pode compartilhar os eventos criados nas redes sociais (Twitter e Facebook) que estiverem vinculadas a conta do centro de hemoterapia, oficializando tais campanhas. Todos os eventos criados pelo servidor do setor de captação do hemocentro, são notificados aos usuários que utilizam o aplicativo.

O usuário comum utiliza o componente App criando um perfil ou conectando-se via Facebook. No App, ele cria e modifica eventos de solicitação de doação de sangue, visualiza os eventos criados por hemocentros e as solicitações de doação criadas por outros usuários e compartilha quaisquer eventos em suas redes sociais vinculadas ao aplicativo. Ele também tem acesso a função reportar doação, onde pode inserir a data da última doação realizada e, assim, receber uma notificação quando estiver apto a doar novamente. Além disso, ele acessa informações sobre a doação de sangue, visualiza um fluxo detalhado sobre o processo de doação e verifica o ponto de coleta mais próximo de acordo com a sua localização.

O usuário indireto é aquele que recebe as informações compartilhadas nas redes sociais (atualmente Facebook e Twitter), tanto pelos servidores dos hemocentros quanto pelos usuários comuns. Dessa forma, o usuário chama-se indireto pois recebe informações sem fazer uso de nenhum componente do sistema, apenas seguindo as páginas do hemocentro em sua rede social de preferência ou através do compartilhamento de eventos realizado pelos amigos de sua rede. Desse modo, a disseminação de informações é atingida por um maior número de pessoas.

\subsection{Implementação dos Componentes}

O desenvolvimento do sistema foi projetado para ser fácil de manter e expandir. Cada componente foi construído com o uso de Design Patterns conhecidos e possui interfaces para os demais componentes, tornando-os mais independentes. Neste artigo fornecemos 
uma visão geral da implementação dos componentes. Para uma descrição mais extensa, acesse o relatório técnico, disponível em https://goo.gl/kkjz3W.

O componente Server separa acesso ao banco, acesso aos serviços e componentes básicos usando três camadas: Data Access Object (DAO), Services e Plain Old Java Objects (Pojos). DAO generaliza o acesso ao banco, permitindo integração com diferentes sistemas sem a necessidade de adaptar o restante do código. Services generaliza o acesso aos serviços prestados, permitindo que os componentes App e Web se integrem com facilidade. Pojos compreende funcionalidades comuns que não dependem da herança de interfaces, facilitando a manutenção e tornando suas instâncias "genéricas" para todo o programa.

O componente App separa interface com o usuário, controle sobre a execução dos serviços e acesso às informações com quatro camadas: View, Controller, Model e DAO. View captura todas as ações do usuário e interage com Controller. Controller administra as ações do usuário, resolvendo-as localmente ou interagindo com o servidor de acordo com a necessidade. Ele pode também receber informações do servidor via Pushing. Models equivale a camada POJOS do componente Server, visto que nela estão todos os componentes básicos. DAO é utilizada por Controller para acessar os dados. Ela abstrai a comunicação com o servidor. Similarmente ao caso do componente Server, todos as camadas podem evoluir de forma bastante independente.

O componente Web também utiliza o padrão Model-View-Controller (MVC). Assim como em App, Model e View fornecem, respectivamente os componentes básicos e a interface com usuário. Controller interpreta as ações do usuário que são enviadas para a Model e/ou para a View, direcionando todo o fluxo de informação passado pelo sistema. Ele também coordena a interação com o componente Server, realizada pela interface REST. Com o objetivo de oferecer uma experiência agradável e intuitiva ao usuário, foi utilizado o conceito de Material Design [Google 2014]. Isso permite fornecer uma interface adaptável a celulares, tablets e computadores de mesa.

\section{Aspectos de avaliação}

Para validar o protótipo inicial do sistema, serão realizados estudos de caso com usuários do sistema. Especificamente, pretende-se realizar uma avaliação funcional com o HEMORGS (Hemocentro do Estado do Rio Grande do Sul), o qual demonstrou interesse na utilização da ferramenta e comprometeu-se na realização dos testes do sistema e no suporte à manutenção das informações sobre a doação de sangue. Para isso, pretende-se formalizar um caso de experimentação do sistema com os servidores do setor de captação de doadores do hemocentro, verificando suas satisfações com relação aos seguintes tópicos: avaliação das funcionalidades, avaliação da interface e efeitos do uso do sistema.

Para realizar a avaliação das funcionalidades, cada serviço prestado pelo componente $\mathrm{Web}$ será listado e avaliado conforme seu desempenho frente às necessidades do hemocentro. Essa avaliação será dada através de um sistema de pontuação, de 0 (não 
satisfaz a necessidade) a 5 (satisfaz a necessidade), para cada funcionalidade e um campo para preenchimento de observações.

A avaliação da interface qualificará o processo de comunicação do sistema com o usuário. Neste item será exposto o grau de dificuldade no uso de cada serviço disponível no componente $W e b$ a partir de um sistema de pontuação de 0 (muito difícil) a 5 (fácil). Dessa forma, será possível qualificar o uso do software e identificar os problemas de interação.

Além disso, serão produzidos questionários a fim de analisar a aceitação do componente $A p p$ a partir dos conceitos de avaliação da interface e avaliação das funcionalidades citadas anteriormente. Para tal, será utilizado o "Modelo de Aceitação de Tecnologia (Technology Aceptance Model - TAM)". O modelo TAM foi projetado para compreender a relação causal entre variáveis externas de aceitação dos usuários e o uso real do computador, buscando entender o comportamento destes usuários através do conhecimento da utilidade e da facilidade de utilização percebida por eles [Silva et al. 2012]. Em outras palavras, o modelo considera dois aspectos: (1) o desempenho da tecnologia quanto as percepções do usuário; e (2) o esforço que o usuário terá de fazer para usufruir do sistema.

Para avaliar os efeitos do uso do sistema, será analisada a taxa de conversão. Especificamente, serão monitoradas métricas relacionadas a aceitação dos doadores e a qualidade de doadores pré e pós implantação do sistema. Essas medidas serão separadas em três categorias: (1) alcance do compartilhamento de eventos nas redes sociais, (2) conhecimento do doador sobre o sistema e (3) comparação dos registros de doações no aplicativo e no hemocentro. A categoria (1) analisará informações sobre as publicações dos eventos como quantidade de cliques, visualizações e alcance de usuários indiretos através das ferramentas estatísticas das próprias redes. A categoria (2) será realizada através de um questionário no hemocentro a fim de saber como o doador soube da necessidade de doação, se ele conhece o sistema e se utiliza ou passaria a utilizar sabendo de sua existência. Na categoria (3), os registros de doações no aplicativo, no hemocentro, antes e após aplicação do sistema, serão comparados a fim de verificar se o uso do sistema quanto a captação de doadores foi satisfatório.

\section{Conclusões e trabalhos futuros}

O presente trabalho descreve um sistema projetado para auxiliar na captação de doadores de sangue. Dessa forma, a principal contribuição do trabalho é a proposta de uma plataforma que auxilie a diminuição de um problema relevante na sociedade por meio de uma tecnologia social. As principais funcionalidades oferecidas são o acesso fácil à informações pautadas [Ministério da Saúde 2013, 2016], ampliação dos papéis de usuários e servidores de hemocentros na criação de conteúdo e a disseminação mais abrangente (via redes sociais, permitindo inserção de usuários de forma indireta). 
Dentre as melhorias já compreendidas para o aplicativo, visa-se a desenvolver um sistema de reputação e controle de acesso para a criação, aprovação e remoção de eventos. Pretende-se também fazer uma série de testes funcionais com possíveis usuários do aplicativo e usuários do setor de captação do HEMORGS, para que assim seja possível a implantação do sistema no hemocentro do Rio Grande do Sul e, posteriormente, a implantação deste nos centros de coleta pública do Brasil. Como objetivo final, busca-se oferecer a obtenção de um Sistema de auxílio e motivação para doação de sangue que atenda toda a população.

\section{Referências}

Dani, L. (2009). A Doação de Sangue no Contexto do Grupo Hospitalar Conceição. ICITC, Fundação Oswaldo Cruz.

Fowler, James H., e Christakis, Nicholas A. (2010). Cooperative behavior cascades in human social networks. Proceedings of the National Academy of Sciences, 107.12.

Giacomini, L. (2007). Elementos para a organização do trabalho em hemoterapia com vistas à fidelização do doador voluntário de sangue. Programa de Pós-Graduação em Enfermagem, Universidade Federal do Rio Grande (FURG).

Google (2014). Material Design. Disponível em: <https://material.io/guidelines/\#> Acesso em: 04 de mar. 2017.

Gutiérrez, M. G., TEJADA, E. S., e CRUZ, J. R. (2003). Estudio de factores socioculturales relacionados con la donación voluntaria de sangre en las Américas. Rev. Panam. Salud Publica, v. 13, n. 2/3, 2003.

Instituto das Cidades Inteligentes. (2017). Hemogram. Disponível em: $<$ https://play.google.com/store/apps/details?id=br.org.curitiba.ici.hemogram\&hl=pt_ BR> Acesso em: 25 de mar. 2016.

Ludwig, S. (2010). Contribuições para a efetividade da comunicação da doação de sangue a partir de uma abordagem persuasiva. Pontifícia Universidade Católica do Rio Grande do Sul (PUCRS).

Pimentel, M., Gerosa, M. A., Filippo, D., Raposo, A., Fuks, H. e de Lucena, C. J. P. (2006). Modelo 3C de colaboração para desenvolvimento de Sistemas Colaborativos. Anais do III Simpósio Brasileiro de Sistemas Colaborativos, pp. 58-67.

Pinochet, L. (2011). Tendências de Tecnologia de Informação na Gestão da Saúde. O Mundo da Saúde, 35(4).

Rodrigues, A. (2015). Hemoliga. Disponível em: $<$ https://play.google.com/store/apps/details?id=br.com.hemoliga\&hl=pt_BR>. Acesso em: 25 mar. 2017. 
Rodrigues, L. L. (2013). Elementos Motivacionais para a Doação de Sangue. Programa de Pós-Graduação em Saúde Coletiva, Universidade de Brasília.

Rodrigues, R. S. M. e Reybnitz, K. S. (2011). Estratégias de captação de doadores de sangue: uma revisão integrativa da literatura. Texto \& Contexto - Enfermagem, 20(2)

Ministério da Saúde (2013). Técnico em hemoterapia: livro texto. Ministério da Saúde, Secretaria de Gestão do Trabalho e da Educação na Saúde, Departamento de Gestão da Educação na Saúde - Brasília: Ministério da Saúde, 2013.

Ministério da Saúde (2014). Ministério alerta para a redução no estoque de sangue. Disponível em: <http://www.brasil.gov.br/saude/2012/05/ministerio-alerta-parareducao-no-estoque-de-sangue $>$. Acesso em: 11 nov. 2015.

Ministério da Saúde (2016). Gabinete do Ministro. Portaria 158 de 04 de fevereiro de 2016. Redefine o regulamento técnico de procedimentos hemoterápicos. Diário Oficial da União; Poder Executivo, de 05 de fevereiro de 2016. Seção 1, p. 37

Silva Junior, J. B., Costa, C. S. e Baccara, J. P. A. (2015). Regulação de sangue no Brasil: contextualização para o aperfeiçoamento. Rev Panam Salud Publica, 38(4)

Silva, P. C., Pimentel, V. e Soares, J. (2012). A utilização do computador na educação: aplicando o technology acceptance model (TAM). Biblionline, 8(esp)

Underline. (2016). Hemobile. Disponível em: $<$ https://play.google.com/store/apps/details?id=com.hemobile\&hl=pt $>$. Acesso em: 25 mar. 2017.

World Health Organization (WHO) (2015). Model lists of essential medicines. WHO. Disponível em: < http://www.who.int/medicines/publications/essentialmedicines/en/> Acesso em: 04 de abr. 2017.

World Health Organization (WHO) (2006). Blood safety and donation. WHO. Disponível em: $\quad<$ http://www.who.int/bloodsafety/global_database/GDBSFactSheet\%20.pdf $>$ Acesso em: 06 de abr. 2017. 